\section{Nationale Leitlinien jetzt auch per App verfügbar}

Die "Nationalen Versorgungsleitlinien" (NVL) können sich Ärzte jetzt auch per App auf iPhone oder iPad ansehen. Das geht über die App „VersorgungsLeitlinien" des Ärztlichen Zentrums für Qualität in der Medizin (ÄZQ), die kostenfrei im iTunesStore (ihttp://itunes.apple.com) heruntergeladen werden kann. Nutzer finden in der App die vollständige Kurzfassung der Versorgungsleitlinien - inklusive aller Handlungsempfehlungen, erläuternden Hintergrundtexten und Übersichtstabellen.

Die App ist auch für das Patientengespräch interessant. Sie enthält spezielle Patienteninformationen, genauer werden kurz und für Patienten verständlich Erkrankungen erklärt und es wird auf weiterführendes Info-Material verwiesen. Bislang sind allerdings nur die NVL zu Asthma, Kreuzschmerz und Herzinsuffizienz verfügbar. Nach und nach sollen aber alle NVL für die App aufbereitet werden, meldet das ÄZQ. Dabei steht das Programm für Nationale Versorgungsleitlinien unter der Trägerschaft von Bundesärztekammer, Kassenärztlicher Bundesvereinigung und der Arbeitsgemeinschaft der Wissenschaftlichen Medizinischen Fachgesellschaften. Das ÄZQ wurde allerdings mit der Umsetzung der Leitlinien beauftragt.

\section{medatixx und samedi legen} Online-Kalender auf

Der Arztsoftware-Anbieter medatixx bietet seinen Kunden seit 1. Oktober einen Online-Terminkalender an. Der webbasierte Kalender „ixx.time powered by samedi" ist ein gemeinschaftliches Projekt mit dem Web-Software-Anbieter samedi, der bereits seit Jahren ein Online-Terminbuchungssystem für Praxen bereitstellt. Über den Online-Kalender kann laut medatixx nicht nur die Termin-Kommunikation mit Patienten, sondern auch mit anderen Praxen und Kliniken laufen.

Zum Start des Online-Kalenders bietet medatixx Ärzten eine besondere Aktion: Um die Wartezeit auf den neuen Kalender zu verkürzen, würden die ersten $\mathbf{5 0 0}$ Interessenten, die perE-Mail an info@ medatixx.de Informationen anfordern oder via Kommentar auf facebook.de/ medatixx oder per \#ixxtime-Twitternachricht @medatixx ihr Interesse signalisieren, gratis einen ixx.time-Lichtwecker erhalten.
Die "Just in Time-Funktion“ bestellt den Patienten in die Praxis, wenn er auch wirklich an der Reihe ist. Der Patient kann bei der Terminvereinbarung seinen Standort angeben, das System kalkuliert dann die Zeit und gibt dem Patienten rechtzeitig Bescheid, wann er sich auf den Weg zum Arzt machen soll.

Eine weitere Entlastung für das Praxispersonal ist laut Adendorff der „Wartezimmer-Organizer“. Ein Monitor im Wartezimmer gibt den Patienten Bescheid, in welches Behandlungszimmer sie zu gehen haben. Auch kennt das System den Grund für den Arztbesuch und kann so die Zeit für die Behandlung kalkulieren. „Die Information zu Praxiszimmer, Dauer der Behandlung und ähnliches muss einmalig bei der Installation festgelegt werden, danach arbeitet das System alleine“, erklärt Adendorff.

Das „Sprechzimmermodul“ informiert den Arzt in welchem Zimmer sich der nächste Patient befindet, wer er ist und was der Grund für seinen Besuch ist.
Adendorffs Ziel war, dass mit AJITS ${ }^{\curvearrowleft}$ Arzt und MFA wieder mehr Zeit für die Patienten haben. „Auch fallen dadurch Überstunden weg, die Mitarbeiter kommen pünktlich nach Hause, was die $\mathrm{Zu}$ friedenheit im Team steigert. "Laut Adendorff spart das System der MFA zwei und dem Arzt eine Stunde Zeit am Tag.

AJITS ${ }^{\circledR}$ funktioniere nicht nur in Einzelpraxen, sondern auch in Gemeinschaftspraxen, MVZ und Kliniken, so der Entwickler. Es können mehrere Ärzte und auch Wartezimmer in das System eingepflegt werden. „Es ist sehr flexibel und mit jedem Praxissystem kompatibel“, sagt Adendorff. Die Kosten für eine Einzelpraxis belaufen sich auf etwa $2.000 €$ für alle fünf Module. Es lassen sich aber auch nach Bedarf einzelne Module kaufen.

Adendorff hat mittlerweile auch schon die nächsten Ideen. Bei seiner Recherche in der Hausarztpraxis ist ihm aufgefallen, wie lange die Abrechnung dauert. „Hier kann man auch noch einiges optimieren“, ist er sich sicher. Kerstin Mitternacht

\title{
Ältere Generation entdeckt die Vielfalt des Webs für sich
}

\section{Jeder dritte Bundesbürger der Altersgruppe 65 plus ist online aktiv.}

mmer mehr Bundesbürger der Altersgruppe 50 bis 64 Jahre und der Altersgruppe 65 plus tummeln sich im World Wide Web. Das zeigt eine repräsentative Studie des Hightech-Verbands Bitkom (Bundesverband Informationswirtschaft, Telekommunikation und neue Medien) unter mehr als 1.000 Bundesbürgern ab 14 Jahren. Aktuell seien nahezu drei Viertel (74\%) der 50- bis 64-Jährigen im Internet unterwegs, 2011 waren es noch $68 \%$ gewesen. Und immerhin auch jeder Dritte der Altersgruppe 65 plus nutzt regelmäßig das Web. Im Vorjahr war es lediglich jeder Vierte. Der kräftige Anstieg bei der Internetnutzung in dieser Altersgruppe zeige, dass das Internet gerade älteren Menschen viel zu bieten habe, kommentiert Bitkom-Hauptgeschäftsführer Dr. Bernhard Rohleder das Ergebnis. Dabei seien $41 \%$ der Generation 65 plus, die über einen Internetanschluss verfügt, auch in sozialen Netzen wie Facebook aktiv. Bitkom erwartet, dass sich letzte- $S O C I A L$ MEDIA rer Trend weiter fortsetzt. Der Grund: Insbesondere neue Geräte wie Tablet Computer, die einfach zu bedienen seien, würden helfen, die für Senioren bestehenden Hürden auf dem Weg ins Internet abzubauen.

Dass in der Altersgruppe 14 bis 29 Jahren mit $98 \%$ nahezu jeder Bundesbürger das Internet nutzt, überrascht hingegen nicht. Das war 2011 schon ähnlich. Bei den 30- bis 49-Jährigen geben nun aber bereits $90 \%$ an, regelmäßig im World Wide Web unterwegs zu sein.

Insgesamt sind laut der Studie bundesweit übrigens drei Viertel der Privatpersonen regelmäßig online. Rebekka Höhl 